\title{
Differential Effects of Neurotrophins and Schwann Cell-Derived Signals on Neuronal Survival/Growth and Synaptogenesis
}

\author{
H. Benjamin Peng, ${ }^{1,2}$ Jie-Fei Yang, ${ }^{3}$ Zhengshan Dai, ${ }^{2}$ Chi Wai Lee, ${ }^{1}$ Hiu Wai Hung, ${ }^{1}$ Zhi Hua Feng, ${ }^{3}$ and Chien-Ping Ko ${ }^{3}$ \\ ${ }^{1}$ Department of Biology, Hong Kong University of Science and Technology, Kowloon, Hong Kong, ${ }^{2}$ Department of Cell and Developmental Biology, \\ University of North Carolina, Chapel Hill, North Carolina 27599-7090, and 3 Section of Neurobiology, Department of Biological Sciences, University of \\ Southern California, Los Angeles, California 90089-2520
}

Recent studies have shown that the survival of mammalian motoneurons in vitro is promoted by neurotrophins (NTs) and cAMP. There is also evidence that neurotrophins enhance transmitter release. We thus investigated whether these agents also promote synaptogenesis. Cultured Xenopus spinal cord neurons were treated with a mixture of BDNF, glia-derived neurotrophic factor, NT-3, and NT-4, in addition to forskolin and IBMX or the cell-permeant form of cAMP, to elevate the cAMP level. The outgrowth and survival of neurons were dramatically increased by this trophic stimulation. However, when these neurons were cocultured with muscle cells, the trophic agents resulted in a failure of synaptogenesis. Specifically, the induction of ACh receptor (AChR) clustering in cultured muscle cells was inhibited at nerve-muscle contacts, in sharp contrast to control, untreated cocultures. Because AChR clustering induced by agrin or growth factor-coated beads in muscle cells was unaffected by trophic stimulation, its effect on synaptogenesis is presynaptic in origin. In the control, agrin was deposited along the neurite and at nerve-muscle contacts. This was significantly downregulated in cultures treated with trophic stimuli. Reverse transcriptase-PCR analyses showed that this decrease in agrin deposition was caused by an inhibition of agrin synthesis by trophic stimuli. Both agrin synthesis and induction of AChR clustering were restored under trophic stimulation when Schwann cell-conditioned medium was introduced. These results suggest that trophic stimulation maintains spinal neurons in the growth state, and Schwann cell-derived factors allow them to switch to the synaptogenic state.

Key words: neurotrophins; neuromuscular junction; NMJ; agrin; Schwann cell; synaptogenesis; neuronal survival

\section{Introduction}

The development of the nervous system is a complicated, multistep process. From neurogenesis to synaptogenesis, individual neurons encounter a variety of environmental factors that can profoundly influence their growth, differentiation, and survival (Zigmond et al., 1999). Among these factors, the role of the target-derived neurotrophins (NTs) has been particularly well studied. Neurotrophins are obligatory for the survival of sensory neurons and sympathetic neurons (Davies, 1994; Snider, 1994; Thoenen, 1995). Recent studies have also elucidated the essential role of this family of molecules in the survival of motoneurons. The skeletal muscle, as the target tissue of motoneurons, is the source of several neurotrophins, including BDNF, NT-3, NT-4/5, and glia-derived neurotrophic factor (GDNF) (Maisonpierre et al., 1990; Koliatsos et al., 1993; Henderson et al., 1994; Funakoshi et al., 1995). These factors are needed for the survival of spinal motoneurons both in vitro and in vivo (Henderson et al., 1993;

Received 0ct. 10, 2002; revised March 10, 2003; accepted April 2, 2003.

This work was supported by National Institutes of Health Grants NS36754, NS23583 (H.B.P.), and NS17954 (C.P.K.), Research Grants Council Grant HKUST6107/01M (H.B.P.), and a Muscular Dystrophy Association research grant (C.P.K.).

Correspondence should be addressed to either of the following: Dr. H. Benjamin Peng, Department of Biology, Hong Kong University of Science and Technology, ClearWater Bay, Kowloon, Hong Kong, E-mail: penghb@ust.hk; or to Dr. Chien-Ping Ko, Section of Neurobiology, Department of Biological Sciences, University of Southern California, Los Angeles, CA 90089-2520, E-mail: cko@usc.edu.

Z. Dai's present address: Biomachines, 507 Airport Boulevard, Suite 107, Morrisville, NC 27560.

Copyright $\odot 2003$ Society for Neuroscience $\quad$ 0270-6474/03/235050-11\$15.00/0
Hughes et al., 1993; Vejsada et al., 1995; Becker et al., 1998). In addition to promoting neuronal survival, neurotrophins are also involved in neuronal differentiation and axonal outgrowth. This is illustrated by the well known response of pheochromocytoma 12 cells to NGF to initiate neurite outgrowth (Unsicker et al., 1978; Leonard et al., 1988). Thus, neurotrophins define a critical need for the survival and outgrowth of neurons. After outgrowth, neurons establish synaptic connections with their targets. During synaptogenesis, the neurite outgrowth has to stop to allow the establishment of a stable connection between the presynaptic and postsynaptic partners (Sanes and Lichtman, 1999). This is accompanied by the development of synaptic specializations for neurotransmitter release and detection. Thus, the neuron needs to switch from the growth state to the synaptogenic state as it establishes contact with the target. Recent studies have also suggested the role of neurotrophins in modulating synaptic functions and plasticity (Wang et al., 1995, 2002; Klintsova and Greenough, 1999; Seil and Drake-Baumann, 2000). For example, neurotrophins enhance transmitter release at both central and peripheral synapses (Lohof et al., 1993; Carmignoto et al., 1997; He et al., 2000; Poo, 2001). However, it is not clear whether they also define the synaptogenic state of the neuron. In other words, do neurotrophins also serve as synaptotrophins?

The spinal cord neurons isolated from Xenopus embryos offer a convenient system to study neurite outgrowth and synaptogenesis (Cohen, 1980). They respond to bath application of neurotrophins by enhanced survival and outgrowth and to experimen- 
tally generated neurotrophin gradients by directional growth either toward or away from the source of the factor (Ming et al., 1997; Song and Poo, 1999, 2001). When cocultured with myotomal muscle cells, functional neuromuscular junctions (NMJs) that develop to a high degree of maturity are formed (Anderson et al., 1977). In an attempt to promote the formation of these NMJs in vitro, we experimented with the four neurotrophins described above, which are known to be synthesized by skeletal muscle cells. Because the elevation of intracellular cAMP level has been shown to be sufficient for motoneuron survival (Hanson et al., 1998), we also experimented with its effect on these Xenopus neurons. Consistent with previous results, we found that these neurotrophins and CAMP, applied either singly or in combination, indeed enhanced the growth and survival of Xenopus spinal cord neurons. To our surprise, this treatment rendered these neurons refractory to synaptogenesis, as shown by the absence of postsynaptic acetylcholine receptor (AChR) clustering at the nerve-muscle contacts, a hallmark of NMJ development. The postsynaptic development is triggered by the secretion of neural agrin from the motor axon, which activates the muscle-specific receptor tyrosine kinase (MuSK) (McMahan, 1990; Glass et al., 1996; Sanes and Lichtman, 2001). We thus investigated the pattern of agrin deposition and transcription in response to neurotrophin treatment. We report here that agrin synthesis and deposition are downregulated by trophic stimulation of spinal neurons. Recent studies have suggested that Schwann cells may promote synaptic regeneration and formation (Son and Thompson, 1995a,b, 1996; Herrera et al., 2000; Koirala et al., 2000), as well as enhancing the aggregation of AChRs (Yang et al., 2001). We thus examined whether factors released by Schwann cells might promote synaptogenesis in the nerve-muscle coculture. The present results show that Schwann cell-conditioned medium (SCCM) is able to restore the NMJ formation and neuronal agrin synthesis after trophic stimulation. This study thus delineates a novel regulation of synaptogenesis by neurotrophins and Schwann cell-derived factors. An elevated level of neurotrophins maintains the motoneuron in the growth state even after its axons come into contact with the target muscle. Synaptogenesis may require Schwann cell-derived factors that promote agrin secretion and postsynaptic development.

\section{Materials and Methods}

\section{Reagents}

The entactin-collagen IV-laminin cell attachment matrix was purchased from Upstate Biotechnology (Waltham, MA). Neurotrophins (BDNF, NT-3, NT-4/5, and GDNF) were obtained from Invitrogen (San Diego, CA). Chlorophenylthio (CPT)-cAMP, Rp-cAMP, forskolin, IBMX (3isobutyl-1-methylxanthine), and K-252a were obtained from Sigma (St. Louis, MO). Tetramethylrhodamine-conjugated $\alpha$-bungarotoxin (RBTX) was obtained from Molecular Probes (Eugene, OR).

\section{Spinal neuron culture and neuron survival assay}

The spinal neurons were isolated from stage 22 Xenopus embryos according to a method published previously (Peng et al., 1991). In short, the neural tubes were dissected from embryos and dissociated in $\mathrm{Ca}^{2+}$ - and $\mathrm{Mg}^{2+}$-free Steinberg solution. Isolated neurons were cultured on cover glass coated with entactin-collagen IV-laminin in Steinberg medium. To prepare the nerve-muscle coculture, the myotomes were first isolated from the embryos, dissociated in $\mathrm{Ca}^{2+}$ - and $\mathrm{Mg}^{2+}$-free Steinberg solution, and plated on the culture substratum. After $1-3 \mathrm{~d}$ in culture, spinal neurons were seeded into the muscle cultures. Nerve-muscle contacts could be detected within $1 \mathrm{~d}$ of coculture.

To assess the survival of neurons in culture, phase-contrast microscopy was used to score the number of neuronal cell bodies and the number of neurites remaining at regular intervals after plating. All of the neurons on the cover glass were scored. Cultures were prepared in triplicate, and the mean number was calculated.

To visualize nerve-muscle contacts with fluorescence microscopy, synaptophysin tagged with green fluorescence protein (GFP) was expressed in spinal neurons. GFP-synaptophysin cDNA [kindly provided by Dr. Lian Li (Emory University, Atlanta, GA)] was transcribed in vitro into mRNA using Ambion (Austin, TX) mMESSAGE mMACHINE kits. The mRNA was injected into Xenopus blastomeres at the two-cell stage at 5-10 ng of mRNA per embryo. The neural tubes of the injected embryos were isolated and dissociated to prepare neuron cultures.

\section{Assaying the formation of AChR clusters}

The formation of AChR clusters was assessed by fluorescence microscopy after labeling the nerve-muscle cocultures with R-BTX. The nerve-muscle contacts were determined by phase-contrast microscopy in live cultures. All of the contacts on the cover glass were scored to determine the extent of AChR clustering. In addition to nerve-muscle cocultures, AChR clustering was also examined in pure muscle cultures after the presentation of beads and agrin. Polystyrene latex beads with a $10 \mu \mathrm{m}$ diameter (Polysciences, Warrington, PA) were coated with the growth factor heparin-binding growth-associated molecule [HB-GAM; also known as pleiotrophin; kindly provided by Dr. Heikki Rauvala (University of Helsinki, Helsinki, Finland)] and applied to muscle cells as described previously (Peng et al., 1995). The formation of AChR clusters at bead-muscle contacts was assessed by R-BTX labeling. These beads induce highly localized clustering of AChRs. To induce AChR clustering by agrin, muscle cultures were treated with conditioned medium from human embryonic kidney 293 cells transiently transfected with a cDNA encoding the B8 form of neural agrin (Daggett et al., 1996). Bath application of agrin globally induces AChR clustering throughout the muscle cell. In addition to nerve-induced AChR clustering, the effects of neurotrophins on preexistent AChR clusters (hot spots) were also examined. Hot spots are clusters that are not associated with nerve-muscle contacts and thus do not conform to the neuritic track. They vary in size and shape but are typically $5-20 \mu \mathrm{m}$ in their linear dimensions.

\section{The deposition and transcription of agrin by spinal neurons}

The deposition of agrin along the neurite and the nerve-muscle contact was studied with a polyclonal rabbit antibody, Pab 36 (Godfrey, 1991; Cohen et al., 1994) [a generous gift of Dr. Earl Godfrey (Eastern Virginia Medical School, Norfolk, VA)]. Cultures prepared on the entactincollagen IV-laminin substratum were labeled with Pab 36 followed by FITC-conjugated goat anti-rabbit secondary antibody, either in the living state or after fixation with $95 \%$ ethanol at $-20^{\circ} \mathrm{C}$. The presence of agrin was then examined by fluorescence microscopy. The agrin protein level in cultured neurons was detected by Western blot using anti-agrin monoclonal antibody C3 (also kindly provided by Dr. Earl W. Godfrey) as described previously (Yang et al., 2001).

Reverse transcriptase (RT)-PCR analysis was performed according to a method published previously (Yang et al., 2001). In short, cells from nerve-muscle cocultures or pure neuron cultures were harvested and total RNAs of these tissues were isolated using QuickPrep total RNA extraction kit (Amersham Biosciences, Arlington Heights, IL). Reverse transcription was performed using oligo-dT primer by first-strand cDNA synthesis kit (Amersham Biosciences). PCR primers flanking the alternative splicing site $\mathrm{B}$ of frog agrin were designed on the basis of the GenBank sequence AF096690: forward, $5^{\prime}{ }_{-574}$ TTT GAC GGA AAG ACT TAC CTG ${ }_{594}-3^{\prime}$, and backward, $5^{\prime}{ }_{-726}$ GGC TTC AGT CTT TAT GCT CAG CTC $_{702}-3^{\prime}$. PCR primers for Xenopus glyceraldehyde 3-phosphate dehydrogenase (GAPDH) were designed on the basis of the GenBank sequence U41753: forward, $5^{\prime}{ }_{-129}$ GTT GCT ATC AAT GAC C ${ }_{144}-3^{\prime}$, and backward, $5^{\prime}{ }_{1019}$ AAG ATC CAC AAC ACG GT $_{1003}-3^{\prime}$. Separated by PAGE, PCR products were visualized by UV transilluminator and imaged with a digital camera.

\section{Conditioned media}

Schwann cell-conditioned medium. Adult Xenopus sciatic nerves were dissected out, and epineurial membranes were removed. Nerve trunks were cut and digested with $0.3 \%$ collagenase and $0.25 \%$ trypsin-EDTA (Invitrogen). Dissociated cells were plated on laminin-1-coated culture 
dishes with culture medium consisting of 45\% Leibovitz's L-15 medium (Invitrogen), 45\% Ringer's solution (in mM: $115 \mathrm{NaCl}, 2 \mathrm{CaCl}_{2}, 2.5 \mathrm{KCl}$, and 10 HEPES; pH 7.4), and 10\% fetal calf serum (Invitrogen). After 1 week in culture, this culture medium was replaced by serum-free medium, L-15/Ringer's solution (1:1, v/v). At the second week in culture, there were $\sim 15,000$ Schwann cells that had grown in each culture dish ( $35 \mathrm{~mm}$ diameter) containing $\sim 2 \mathrm{ml}$ of serum-free culture medium. The Schwann cell-conditioned medium was collected once per week and replenished with the fresh serum-free medium. Only cultures containing $\geq 90 \%$ Schwann cells (the rest were fibroblasts) were used. In the cultures treated with NT plus cAMP plus SCCM, the cultures were first incubated in the normal culture medium with NT plus cAMP for $2 \mathrm{~d}$ and then changed to a medium containing $95 \%$ of SCCM in addition to the NT plus cAMP for 2 more days before the examination. In the control cultures treated with SCCM alone, the cultures were incubated with SCCM for $2 \mathrm{~d}$ before final examination.

Muscle-conditioned medium. Pure embryonic Xenopus muscle cultures were prepared according to the method described above. Dissociated muscle cells were plated on culture dishes coated with laminin-1 (Invitrogen). The culture medium contained 50\% L-15 and 50\% Ringer's solution. Conditioned medium from muscle cultures was collected 1 week after plating.

Fibroblast-conditioned medium. Fibroblast cultures were obtained by enzymatic digestion of the epineurial sheath of the sciatic nerve trunk. Dissociated fibroblast cells were plated on culture dishes coated with laminin- 1 and cultured in medium containing 50\% L-15 and 50\% Ringer's solution. Conditioned medium from fibroblast cultures was collected 1 week after plating.

\section{Results}

\section{Effects of neurotrophins and cAMP elevation on neurite outgrowth and survival}

During the first day in culture, Xenopus spinal cord neurons exhibited vigorous growth on a number of substrata. In this study, neurons were plated on cover glass coated with a mixture of three extracellular matrix molecules, entactin, collagen IV, and laminin, extracted from Engelbreth-Holm-Swarm mouse tumor. This substratum has been shown to support the growth of Xenopus spinal neurons and to allow the deposition of agrin secreted by these neurons (Cohen et al., 1994). Without the supply of trophic molecules, these neurons do not survive long in culture. Using the number of neurites as a marker of neuronal growth and survival and treating that number in day 1 culture as $100 \%$, no neurons were found to survive beyond 5-6 d in culture (Fig. 1). The neuronal survival, as scored by the number of cell bodies, paralleled the neurite counts (data not shown).

The effect of neurotrophins on the survival of these neurons was then tested. First, several neurotrophins that have been shown to be expressed by muscle cells were tested individually. Neurons were incubated with a neurotrophin at a concentration of $10 \mathrm{ng} / \mathrm{ml}$ from the time of plating, and their survival during the culture period was assessed (Hanson et al., 1998). As shown in Figure 1, BDNF caused a large increase in neuronal survival. Other neurotrophins also significantly enhanced neuronal survival, but their effects were not as strong as BDNF (data not shown). The effect of cAMP that is sufficient to promote the survival of mammalian spinal motor neurons (Hanson et al., 1998) was also assessed in the Xenopus spinal neuron culture. As shown in Figure 1, elevation of intracellular cAMP by treating neurons with forskolin $(10 \mu \mathrm{M})$, an adenylate cyclase activator, and IBMX $(100 \mu \mathrm{M})$, a phosphodiesterase inhibitor, also resulted in a large increase in neuronal survival. A similar result was seen when CPT-cAMP (125 $\mu \mathrm{M})$, a membrane-permeant form of cAMP, was used instead of forskolin and IBMX (data not shown). The efficacy of cAMP and neurotrophins in promoting spinal

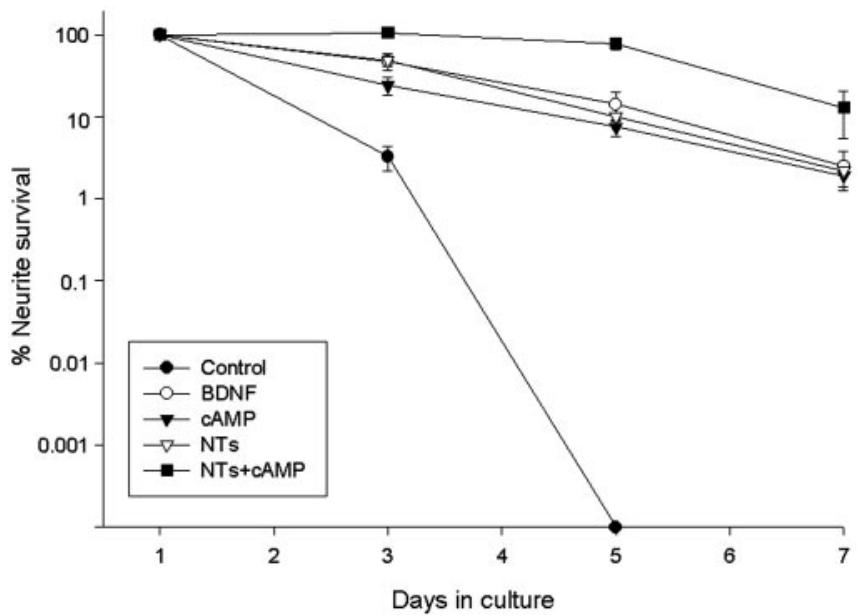

Figure 1. The effect of trophic stimuli on the survival and growth of spinal neurons. The number of neurites was scored as an indicator of neuronal survival and growth. Neurons cultured in the absence of trophic stimuli (control; filled circles) did not survive beyond day 5 in culture. The addition of a single neurotrophin (BDNF; open circles) or a combination of four neurotrophins (NTs; BDNF, NT-3, NT-4/5, and GDNF; open triangles) enhanced neuronal survival and growth. This effect was also seen when intracellular CAMP was elevated by forskolin and IBMX (cAMP; filled triangles). Combining neurotrophins and CAMP (NTs + cAMP; filled squares) further promoted survival and growth. For each point, triplicate cultures, each with at least 100 neurons at day 1 , were scored. A survival of $100 \%$ refers to the number of neurites at day 1 . SEs (error bars) were shown for the data points.

neuron survival was rated as follows: $\mathrm{cAMP} \approx \mathrm{BDNF}>\mathrm{NT} 4 \approx$ GDNF $>$ NT3. A mixture of these four neurotrophins produced the same rescue effect as CAMP or BDNF alone (Fig. 1). However, when neurotrophins were combined with cAMP elevation, the best result in neuronal survival was seen (Fig. 1). With this treatment, little neuronal loss was seen during the first $5 \mathrm{~d}$ in culture. This represents a dramatic increase in neuronal survival over the control. We thus used this combined trophic stimulation in most of our subsequent experiments on synaptogenesis.

\section{Effects of trophic stimuli on synaptogenesis}

When the axons of spinal neurons come into contact with myotomal muscle cells, the formation of the NMJ follows. This is evidenced by the development of AChR clusters at the nervemuscle contact visualized by R-BTX labeling (Anderson and Cohen, 1977). Because the neurite is not always clearly visible when it is in contact with the muscle cell, we expressed GFPsynaptophysin in neurons by blastomere injection of synthetic mRNA and cocultured them with muscle cells isolated from uninjected embryos. As shown in Figure $2 A$, in which the neurite was marked by GFP-synaptophysin, AChRs, labeled by R-BTX, were first clustered into small punctate aggregates along the nerve after the first day of coculture. These aggregates then became consolidated into larger clusters after 1-2 d (Fig. 2 B). NMJ induction is a highly efficacious process in these cocultures, because AChR clustering could be detected at up to $90 \%$ of nerve-muscle contacts. When cocultures were treated with the neurotrophin mixture plus forskolin and IBMX or neurotrophins plus CPTcAMP, there was a large reduction in NMJ formation despite more numerous nerve-muscle contacts than seen in the control as a result of the enhanced neurite outgrowth. Examples of nerve-muscle contacts devoid of AChR clusters in the presence of the trophic stimuli are shown in Figure 3. In contrast to extensive AChR clustering induced in control cultures, contacts in cultures treated with the NT mixture with or without cAMP showed much 

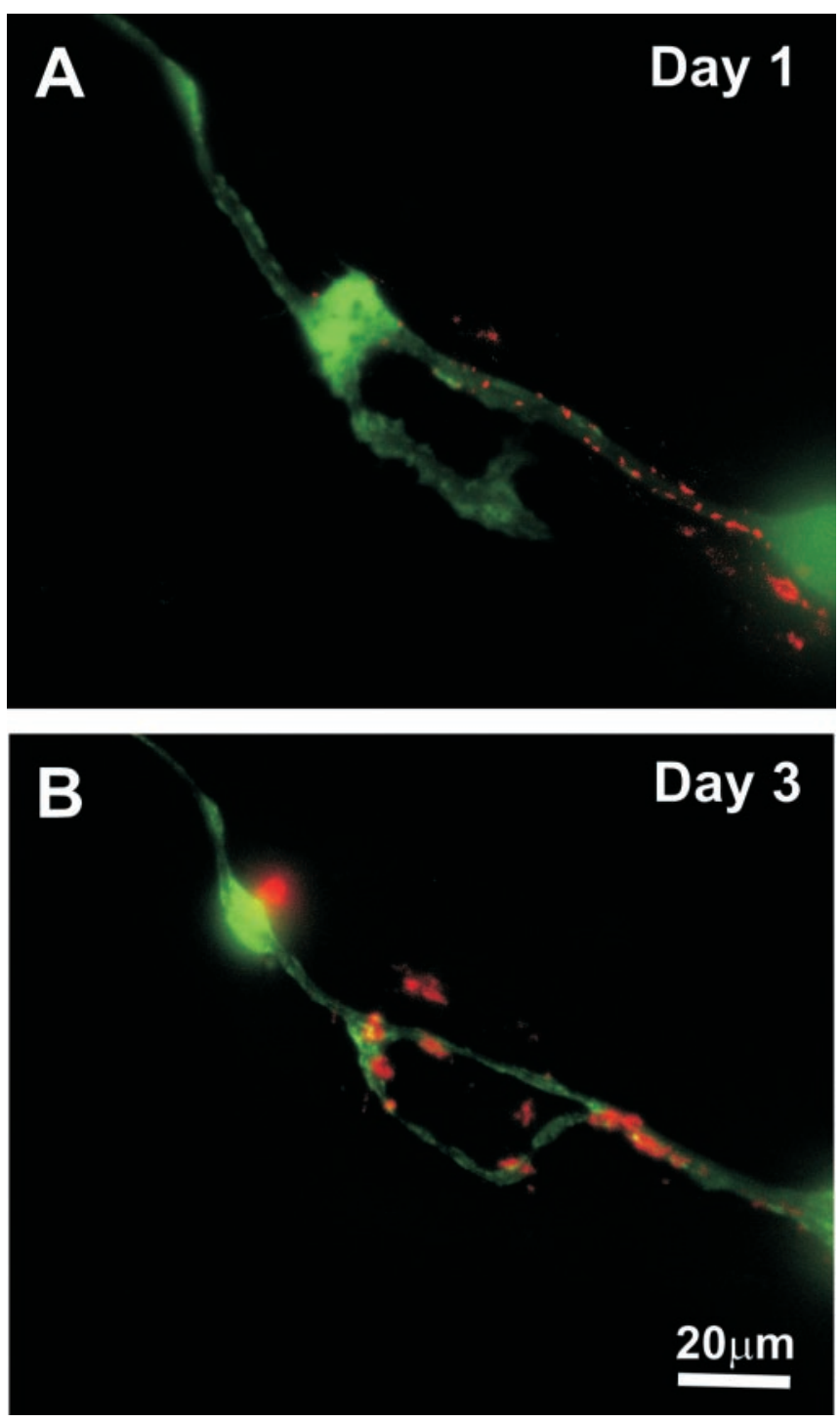

Figure 2. AChR clustering at nerve-muscle contacts. GFP-synaptophysin was used as a presynaptic marker, and R-BTX was used to label postsynaptic AChR clusters. $A, 0$ n day 1 of nervemuscle coculture, punctate $A C h R$ clusters were seen along the contact area. $B$, These clusters were consolidated into larger $A C h R$ patches in day 3. Scale bar: (in $B) A, B, 20 \mu \mathrm{m}$.

reduced postsynaptic specialization as quantified in Figure 4. The neurotrophin effect was reversed by K-252a (100 nM), a specific inhibitor of Trk tyrosine kinase receptors for neurotrophins, but was not reversed by Rp-cAMP $(100 \mu \mathrm{M})$, a membrane-permeant inhibitor of cAMP-dependent protein kinases. A lesser, but significant, inhibition was also seen when cAMP or individual neurotrophins were applied alone (Fig. 4).

In addition to inducing AChR clustering locally along the nerve-muscle contact, innervation also leads to a global effect in the dispersal of preexisting AChR clusters (hot spots) that are often located at a distance away from the contact site (MoodyCorbett and Cohen, 1982; Kuromi and Kidokoro, 1984; Dai and Peng, 1998). Unlike the nerve-induced clusters, hot spots are not associated with the nerve-muscle contacts and are stable in the absence of innervation. Their dispersal was invariably linked to the nerve-induced clustering of AChRs in control cultures (Fig. $3 A, B$ ). Treating cells with neurotrophin and cAMP caused the nerve-contacted muscle cells to retain their hot spots (Fig. $3 D, F$, open arrows). Thus, treatment of cocultures
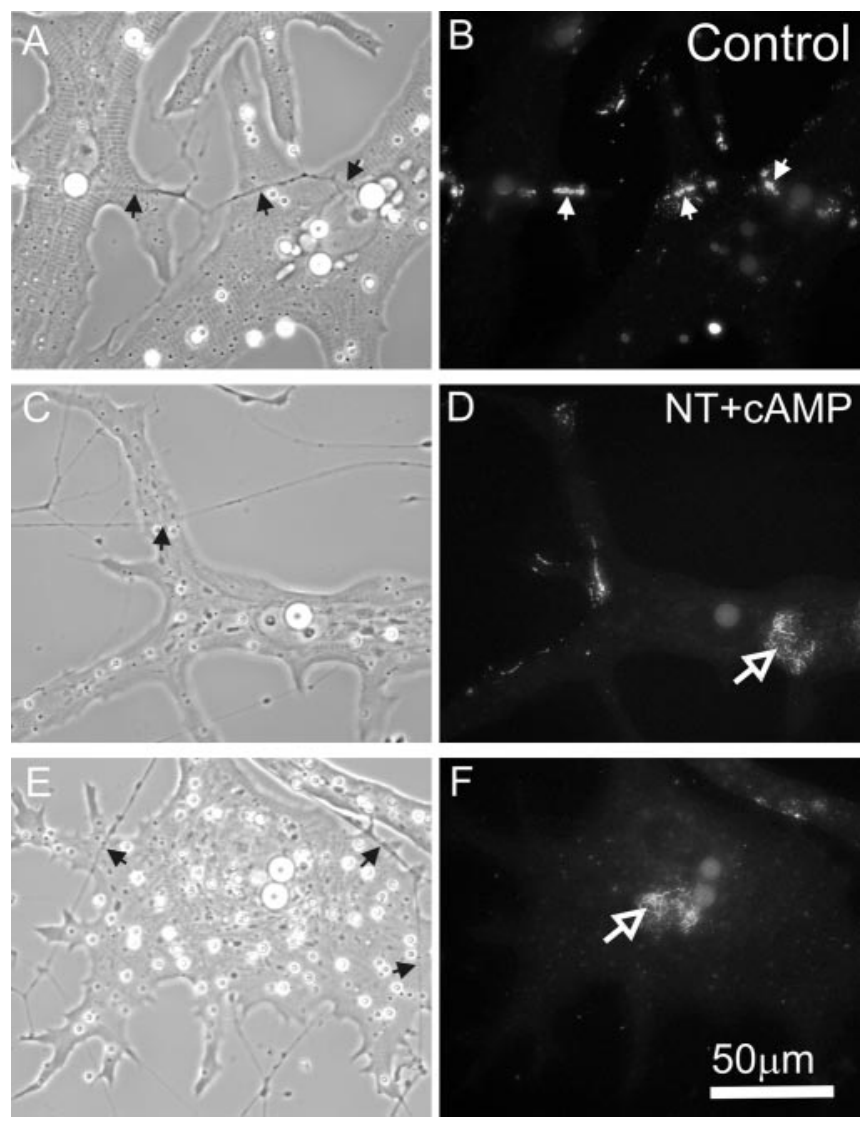

Figure 3. The effect of trophic stimulation on NMJ formation. The formation of AChR clusters (right, white arrows) along nerve-muscle contacts (black arrows in phase images on the left) seen in the control $(A, B)$ was abolished after NT plus cAMP treatment $(C-F)$. However, preexistent AChR hot spots (open arrows) were dispersed in control cocultures but were left intact after trophic stimulation. Scale bar: (in $E) A-F, 50 \mu \mathrm{m}$.

with neurotrophins inhibits both local and global effects associated with synaptogenesis.

To rule out the effect of neurotrophins and cAMP on the ability of muscle cells to cluster AChRs, muscle cultures devoid of neurons were treated with HB-GAM-coated beads or with agrin in the presence of the trophic stimulation. Beads coated with HB-GAM induce focal clustering of AChRs at bead-muscle contacts (Peng et al., 1995). Bath-applied agrin, however, induces global AChR clustering along the entire Xenopus muscle cell (Daggett et al., 1996). Both stimuli induced AChR clusters as in control cultures in the presence of neurotrophins plus cAMP elevation (Fig. $5 A-D$ ) or neurotrophins alone (Fig. $5 E$ ). At individual neurotrophin concentrations of up to $100 \mathrm{ng} / \mathrm{ml}$, the clustering induced by these stimuli was not diminished. Thus, the inhibitory effect of these trophic agents is presynaptic in nature.

\section{Agrin deposition and synthesis}

It is well accepted that the neural agrin secreted by motoneurons is necessary for the induction of postsynaptic specializations at the NMJ (Bowe and Fallon, 1995; Sanes and Lichtman, 2001). To understand the cause of the trophic inhibition on AChR clustering, we examined the deposition of agrin by spinal neurons using Pab 36, a polyclonal antibody that recognizes Xenopus agrin (Godfrey, 1991; Cohen et al., 1994). As shown in Figure 6, $A$ and $B$, the secretion of agrin was easily detected on the substratum along the neuritic tracks in control cultures. The fine structure of the agrin deposition often reflected the neuritic branching pat- 


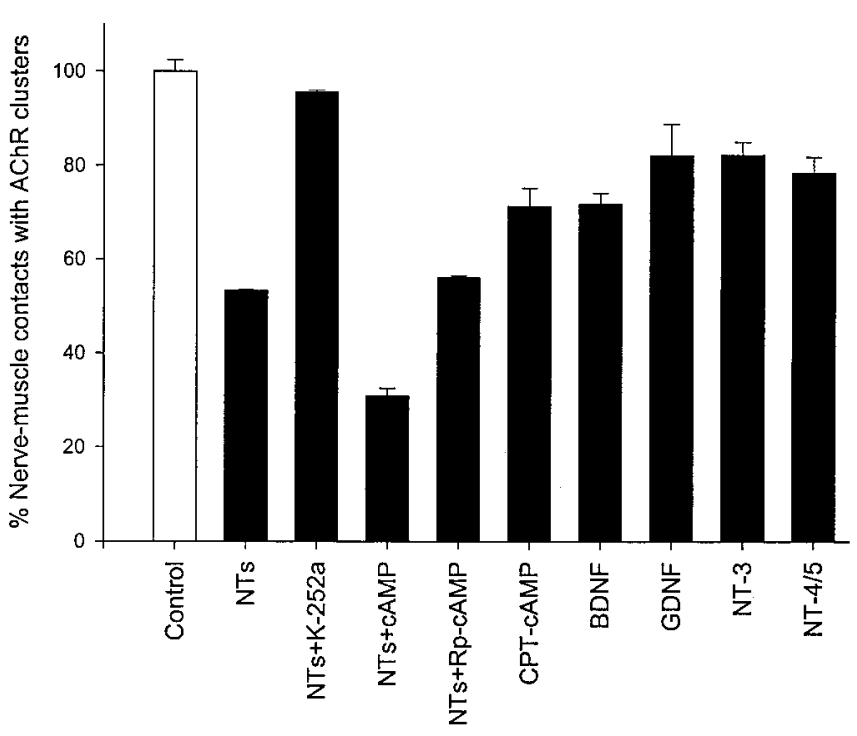

Figure 4. Quantification of AChR clustering at nerve-muscle contacts in response to different stimuli (filled bars). Neurotrophins were used at $10 \mathrm{ng} / \mathrm{ml}$ either singly or in combination. NTs plus CAMP refers to neurotrophins plus forskolin, IBMX, and CPT-CAMP. The extent of AChR clustering was normalized to the control (open bar). Data were collected from three independent experiments with $>160$ nerve-muscle contacts scored. The $p$ values for the difference between the test and the control were as follows: NTs, 0.0002 , NTs plus K-252a, 0.71 ; NTs plus cAMP, 0.0007; NTs plus Rp-CAMP, 0.0003; CPT-CAMP, 0.001; BDNF, 0.001; GDNF, 0.15; NT-3, 0.05 ; and NT-4/5, 0.004. SEs (error bars) are shown.

tern. Trophic stimulation by neurotrophins with or without cAMP, or by cAMP alone, resulted in a dramatic reduction in agrin deposition, as shown by a disappearance or a large reduction of agrin immunoreactivity along the neurites (Figs. $6 C, D, 7$ ). This inhibition was reversed by the Trk inhibitor K-252a but not by the cAMP-dependent protein kinase inhibitor Rp-cAMP (Figs. 6E, F, 7). Individual neurotrophins applied singly also inhibited agrin deposition (Fig. 7). In control nerve-muscle cocultures, agrin was also detected at the contact area (Fig. 6J-K). After trophic stimulation, little agrin deposition was seen along the contact region in parallel with an abolishment of AChR clustering (Fig. 6G-I).

To determine whether trophic stimulation interfered with agrin synthesis, RT-PCR analyses were performed to assess its mRNA levels. Through alternative splicing, four isoforms of agrin that differ in an insert of $0,8,11$, or 19 amino acids at the $B$ site (also known as $\mathrm{Z}$ site in mammalian agrin) near the $\mathrm{C}$ terminal are generated (Tsim et al., 1992). Previous studies have shown that B8, B11, and B19 forms are synthesized by neurons (Tsim et al., 1992) and Schwann cells (Yang et al., 2001), whereas both neurons and non-neuronal cells, including skeletal muscle, express the $\mathrm{B} 0$ form. Whereas neural agrin (B8, B11, and B19) are effective in inducing AChR clustering in muscle cells, B0 agrin is ineffective in bath application assays (McMahan et al., 1992; Gesemann et al., 1995). When neurons were cocultured with muscle cells under normal circumstances, all four forms of agrin transcripts were detected in them (Fig. 8A, lane 1). Consistent with previous results, only the $\mathrm{B} 0$ form was detected in pure muscle cultures (Fig. 8 A, lane 4). Because Schwann cells were not present in this culture system, the B8, B11, and B19 forms of agrin in nerve-muscle cocultures were contributed by neurons only. Upon trophic stimulation by neurotrophins plus cAMP elevation, the expression of neural agrin is downregulated (Fig. $8 \mathrm{~A}$, lane 2). It is noted that $\mathrm{B} 0$ was still present after the trophic
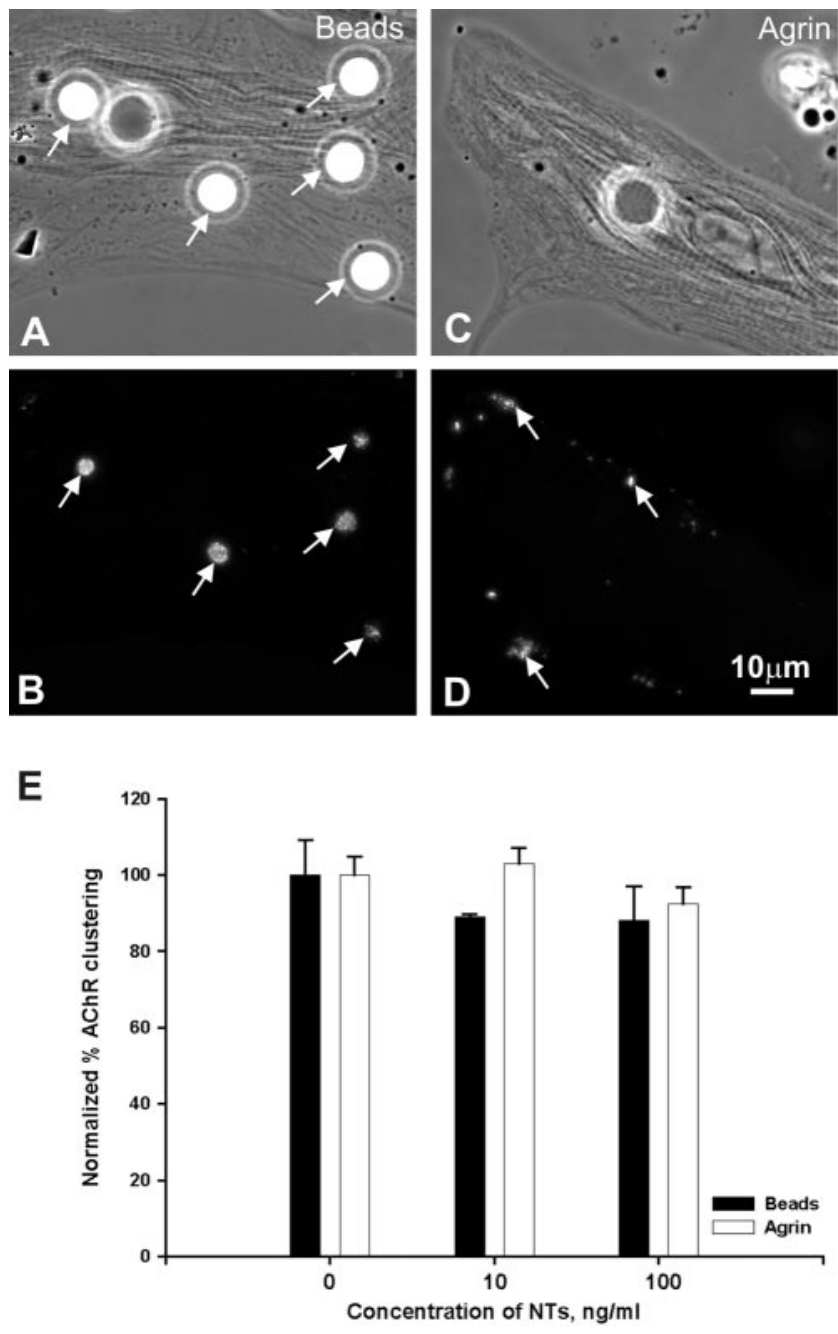

Figure 5. Trophic stimulation by neurotrophins and CAMP did not affect the induction of AChR clustering by HB-GAM-coated beads $(A, B)$ or by agrin $(C, D) . A, B$, Arrows point out correspondence in bead-muscle contacts and $A C h R$ clusters. $D$, Arrows point out several agrininduced clusters. Scale bar: (in D) $A-D, 10 \mu \mathrm{m}$. E, Quantification of $A C h R$ clustering in the presence of all four neurotrophins at different concentrations. For the bead experiments (filled bars), data from four independent experiments with 50 cells at each neurotrophin concentration were pooled. The $p$ values for the difference between treated versus the control were 0.47 (for $10 \mathrm{ng} / \mathrm{ml} \mathrm{NT}$ ) and 0.41 (for $100 \mathrm{ng} / \mathrm{ml} \mathrm{NT}$ ). For the agrin experiment (open bars), 50 cells were scored for each concentration. The mean number of AChR clusters per $100 \mu \mathrm{m}$ of muscle length was measured for each cell. The $p$ values for the difference between treated versus the control were 0.63 (for $10 \mathrm{ng} / \mathrm{ml} \mathrm{NT}$ ) and 0.25 (for $100 \mathrm{ng} / \mathrm{ml} \mathrm{NT}$ ). SEs (error bars) are shown.

stimulation in this nerve-muscle coculture. The presence of B0 is likely of muscle origin (see below). The control sample in which the RNA template was deleted showed no band (Fig. 8 A, lane 5).

To further determine whether the effect of trophic stimulation is directly presynaptic or postsynaptic via muscle, agrin expression in pure neuron cultures was examined. Whereas normal neuron culture showed all four agrin isoforms at the B site as expected (Fig. $8 B_{1}$, lane 1), the trophic stimulation by neurotrophins plus cAMP elevation caused a dramatic decrease in the expression of all of the agrin isoforms, including B0 (Fig. $8 B_{1}$, lane 3 ). Although the agrin expression was greatly downregulated by trophic stimulation, the general gene expression in these neurons was not affected, as indicated by the GAPDH gene expression, which remained unchanged by the treatment (Fig. $8 B_{1}$, bottom panel). This result further suggests that the trophic 

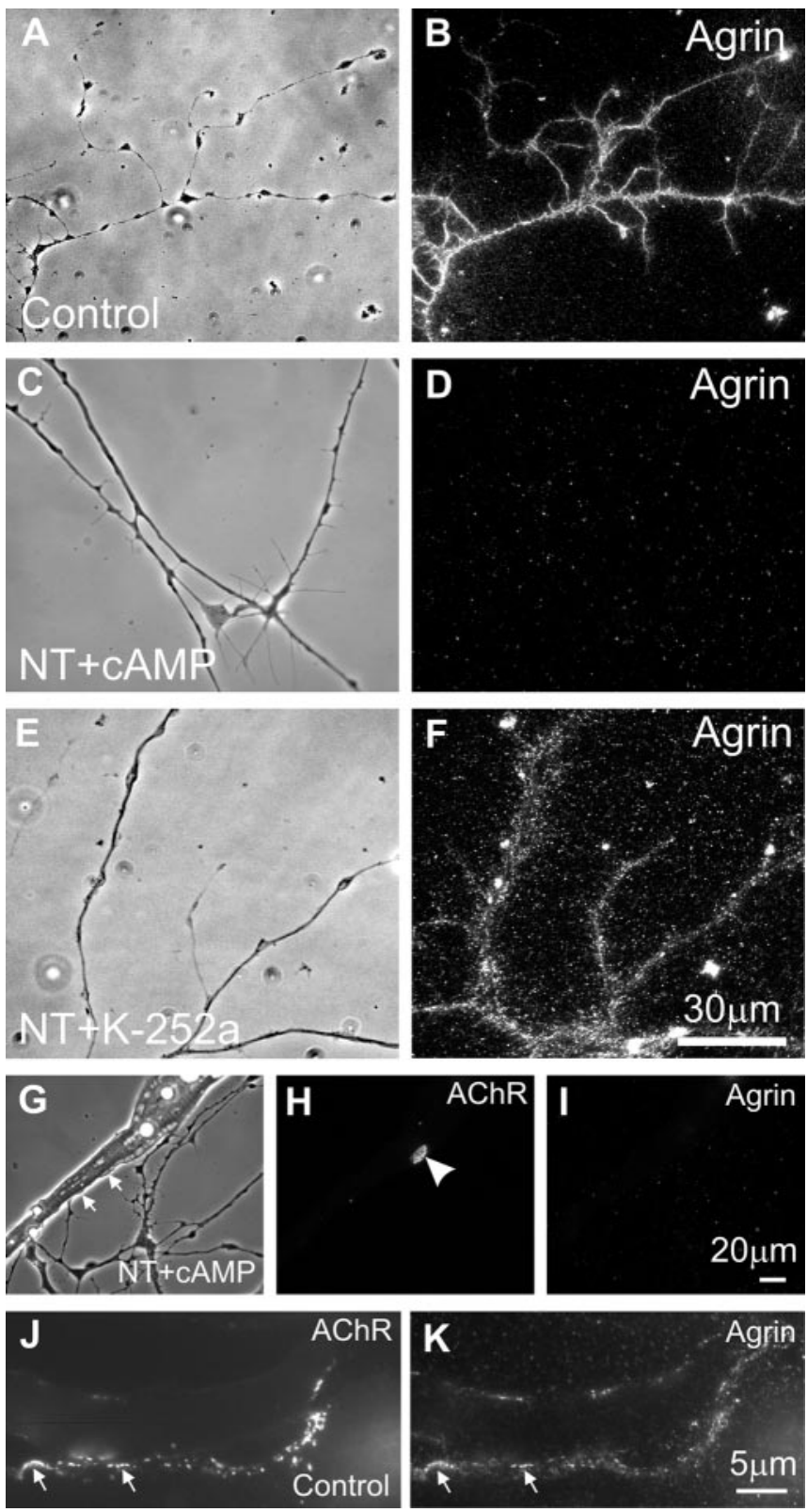

Figure 6. The deposition of agrin along the neurite. $A, B$, Agrin was deposited along the neurite and its branches as revealed by Pab 36 labeling. $C, D$, Agrin deposition was much reduced after trophic stimulation. In this example, there was no association of agrin with the neurites. In the presence of $\mathrm{K}-252 \mathrm{a}$, neurotrophins did not inhibit agrin deposition $(E, F)$. Agrin deposition at nerve-muscle contacts was suppressed by trophicstimulation $(G-l)$. However, an AChR hot spot that was not induced by the nerve was not affected ( $H$, arrowhead). In contrast, nerve-muscle contacts in control cultures were associated with both $A C h R$ clusters and agrin deposition (J, K, arrows). Scale bars: (in $F$ ) $A-F, 30$ $\mu \mathrm{m}$; (in I) G-I, $20 \mu \mathrm{m}$; (in K) J, K, $5 \mu \mathrm{m}$.

stimulation specifically affects the agrin expression in the neurons, but not in muscle.

Together, these two lines of data demonstrate that agrin synthesis and deposition are significantly downregulated after trophic stimulation. This is most likely the cause of the inability of spinal neurons to induce AChR clustering upon trophic influence.

The effect of Schwann cell-conditioned medium in reversing the trophic inhibition

The data presented above suggested that environmental factors determine the state of the neuron between growth and synapto-

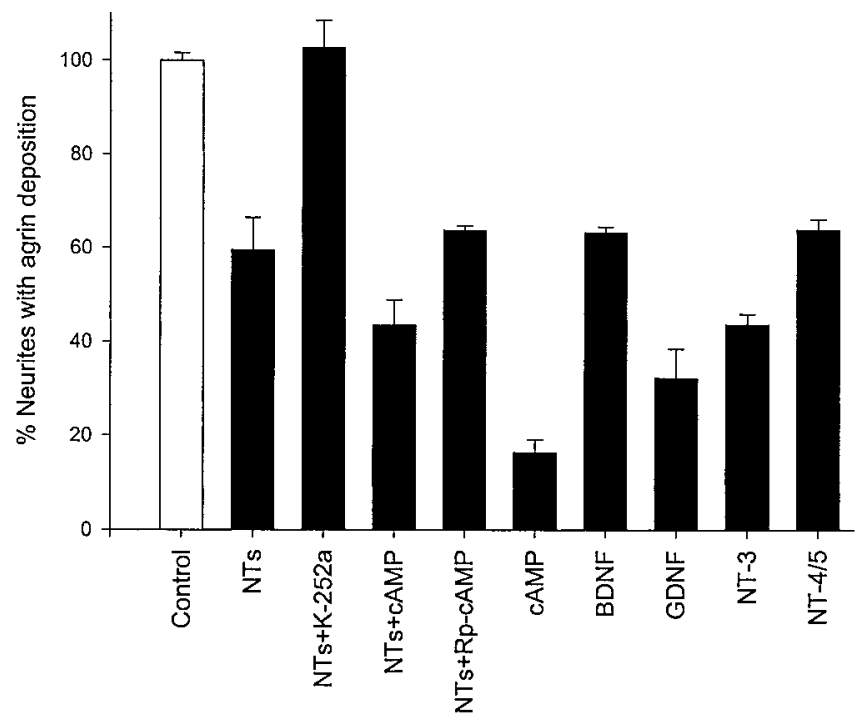

Figure 7. Quantification of agrin deposition in control (open bar) and trophic factorstimulated spinal neuron cultures (filled bars). Data from three independent experiments (except BDNF, NT-3, and NT-4/5) were pooled with $>110$ neurites scored for each treatment. They were normalized to the control. The $p$ values for the difference between the test and the control were as follows: NTs, 0.006, NTs plus K-252a, 0.54; NTs plus CAMP, 0.01; NTs plus Rp-CAMP, 0.0003; CAMP, 0.003; and GDNF, 0.001. Data for BDNF, NT-3, and NT-4/5 were from one experiment only. SEs (error bars) are shown.

genesis as it encounters the target muscle cells. To further understand the molecular determinants that are involved in this switching process, the role of Schwann cell-derived factors was examined. This is based on recent results that Schwann cell processes lead the growth of the axon during terminal sprouting induced by NMJ inactivity (Son and Thompson, 1995b). There is also evidence that synaptogenesis is promoted by glial cells in the CNS (Pfrieger and Barres, 1997). We thus reason that terminal Schwann cells play a role in the transition of the motoneuron from growth to synaptogenic state. This hypothesis was tested in nerve-muscle cocultures with conditioned medium from Schwann cell cultures. In Figure 9, the contrast between trophic stimulation alone and trophic stimulation plus SCCM is shown. Whereas neurotrophins plus cAMP elevation downregulated postsynaptic differentiation (Fig. 9A,B), the addition of SCCM restored to a large extent the ability of spinal neurons to induce AChR clustering (Fig. 9C,D). After SCCM treatment, a fivefold increase in the association of AChR clusters with nerve-muscle contacts was observed (Fig. 9E). In contrast to SCCM, conditioned media from pure muscle or fibroblast culture were ineffective in restoring the reduced AChR aggregation by trophic stimulation (Fig. 9E).

To test whether the restoration of AChR aggregation by the SCCM treatment is attributable to a reversal of the neurotrophininduced suppression of agrin synthesis, RT-PCR analysis was conducted. As shown in Figure 8 A (lane 3), SCCM co-incubation with neurotrophins restored the expression of B8, B11, and B19 neural agrin forms to the control level. Similar restoration was also observed in pure neuron cultures after SCCM treatment (Fig. $8 B_{1}$, lane 4 ), which indicates that SCCM directly affects the agrin expression in neurons. Not only was the gene expression of agrin in neurons restored, a corresponding restoration of agrin protein was also observed with SCCM treatment. As shown in the Western blot in Figure $10 \mathrm{~F}$, agrin immunoreactivity was mostly reduced upon trophic stimulation, compared with normal neu- 
A Nerve-muscle coculture

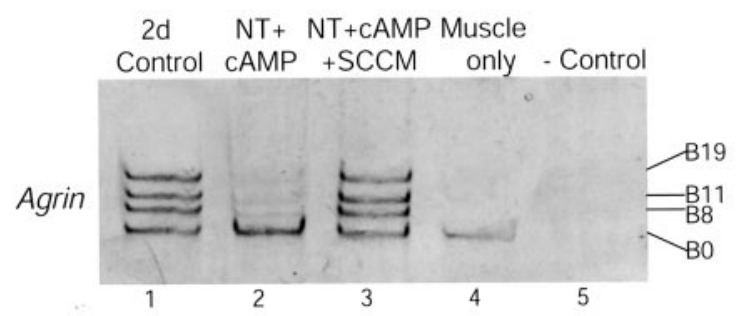

\section{B 1 Pure neuron culture

$\begin{array}{cccc}2 \mathrm{~d} & 2 \mathrm{~d} \text { Control } & \mathrm{NT}+ & \mathrm{NT}+\mathrm{CAMP} \\ \text { Control } & +\mathrm{SCCM} & \text { CAMP } & + \text { SCCM }\end{array}$
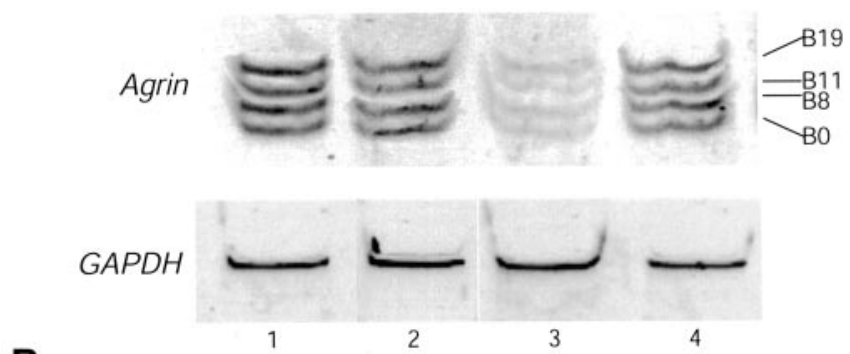

$\mathrm{B}_{2}$

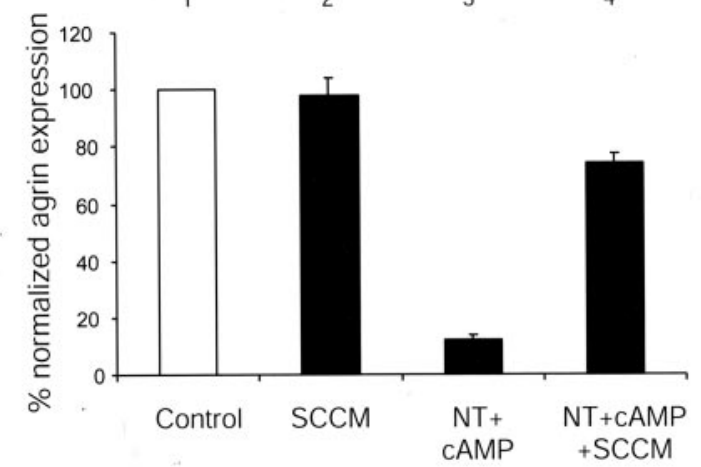

Figure 8. RT-PCR analyses of agrin transcripts. Agrin isoforms at the $B$ splicing site were investigated by RT-PCR analysis in nerve-muscle cocultures $(A)$ and pure neuron culture $(B)$. The nerve-muscle coculture in normal medium at $2 \mathrm{~d}$ showed all four isoforms (inactive $B 0$ and active $B 8, B 11$, and $B 19)(A$, lane 1). The active isoforms were greatly reduced in cultures with NT plus CAMP treatment $(A$, lane 2). The SCCM treatment was able to restore the expression of active isoforms $(A$, lane 3$)$. The $B 0$ isoform observed in the NT plus cAMP treatment probably reflected the muscle agrin expression, as found in pure muscle culture ( $A$, lane 4$)$. No signal was found in RT-PCR reactions containing no RNA sample ( $A$, lane 5$)$. In pure neurons cultured in normal medium for $2 \mathrm{~d}$, all four agrin isoforms were observed ( $B_{1}$, lane 1$)$. SCCM treatment alone in this coculture had no effect on agrin expression $\left(B_{1}\right.$, lane 2). However, all of these agrin is oforms were barely detectable after NT plus CAMP treatment $\left(B_{1}\right.$, lane 3$)$. These agrin isoforms were restored to near-normal level after SCCM treatment $\left(B_{1}\right.$, lane 4$)$. During all of these treatments, the general gene expression in neurons was not affected, as shown by GAPDH gene expression ( $B_{1}$, bottom panel). Agrin expression in pure neurons was quantified by densitometric scanning and normalized for GAPDH expression $\left(B_{2}\right)(n=3$ experiments; data are means \pm SEM). Control, Open bar; experimental, filled bars. These results suggest that the stimulation by neurotrophins can downregulate the agrin expression in neurons, and Schwann cellconditioned medium can reverse this process. They further indicate that the effects of NT plus CAMP and SCCM treatment are presynaptic in origin.

ronal culture (Fig. $10 \mathrm{~F}$, lane 2 vs 1 ). Treatment with SCCM was able to restore the agrin protein level suppressed by trophic stimulation (Fig. 10F, lane 3). Consistent with the expression of agrin gene and protein level, more neurites were found in association with positive agrin staining in cultures treated with neurotrophins, CAMP, and SCCM, compared with reduced agrin staining in cultures treated only with neurotrophins and cAMP (Fig. $10 A-E)$. Although SCCM was effective in restoring the effect of

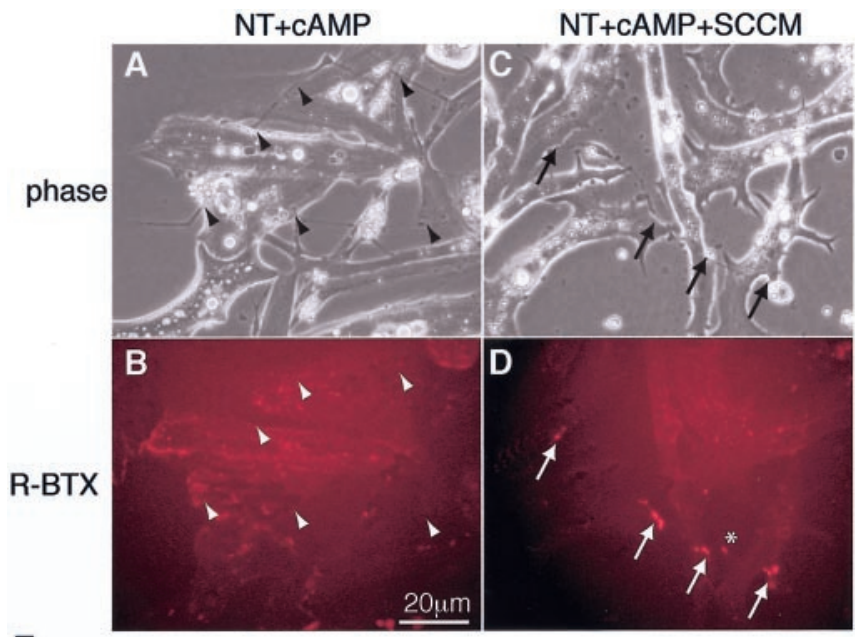

E

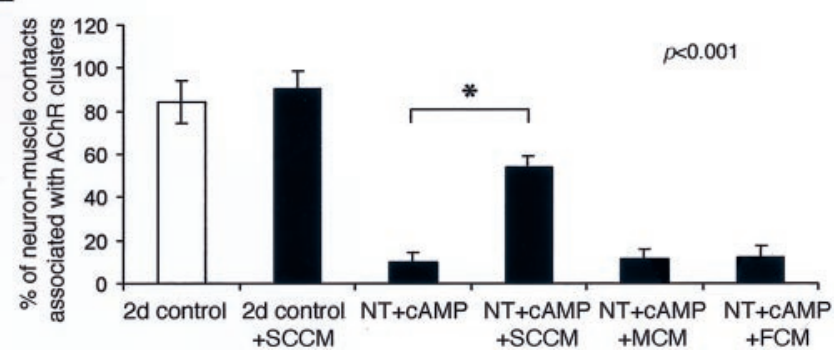

Figure 9. SCCM reversed the effects of trophic stimuli on synaptogenesis. $A, B$, Nervemuscle cocultures were subject to trophic stimulus (NT + cAMP) only. In this example, no R-BTX staining $(B)$ was found on the muscle at the nerve-muscle contacts $(A, B$, arrowheads). Scale bar: (in $B) A-D, 20 \mu \mathrm{m} . C, D$, Nerve-muscle cultures treated with NT plus CAMP plus $S C C M$ showed AChR clusters as revealed by R-BTX staining ( $D$, arrows) at $>50 \%$ nerve-muscle contacts ( $C$, arrows). The asterisk in $D$ marks an AChR hotspot not directly associated with nervemuscle contacts. $E$, The effect of SCCM was quantified. In control nerve-muscle coculture without any treatment, $84.2 \pm 9.8 \%$ of contacts were associated with AChR clusters ( 5 experiments and 130 nerve-muscle contacts counted), and SCCM treatment alone did not change this ratio significantly $(90.5 \pm 8.2 \% ; 5$ experiments and 125 nerve-muscle contacts counted). However, in NT plus CAMP-treated cultures, only $10.1 \pm 6.5 \%$ of contacts showed AChR clusters (5 experiments and 160 nerve-muscle contacts counted). In NT plus CAMP plus SCCM cultures, there was a significant increase in the percentage $(54.2 \pm 7.1 \%)$ of nerve-muscle contacts that showed AChR clusters (5 experiments and 154 nerve-muscle contacts counted) (Student's $t$ test; $\left.{ }^{*} p<0.001\right)$. In contrast, neither muscle-conditioned medium (MCM) nor fibroblastconditioned medium (FCM) reversed the NT plus cAMP effect. Data are means \pm SEM. Open bar, Control; filled bars, experimental.

neurotrophin-induced suppression of synaptogenesis and agrin expression, SCCM by itself did not enhance synapse formation (Fig. 9E) and agrin expression (Fig. $8 B_{1}$ ) under normal condition without trophic stimulation.

These results suggest that, whereas neurotrophins promote neurite survival and outgrowth, SCCM promotes synapse formation, possibly by restoring agrin expression in neurons under trophic stimulation.

\section{Discussion}

Two distinct states in the developmental behavior of spinal neurons have been delineated by trophic stimulation with neurotrophins and cAMP in this study. In the absence of trophic stimulation, these neurons have the propensity to induce synaptogenesis on contact with the target muscle cell. However, upon stimulation, the growth and survival state is favored at the expense of the synaptogenic ability of these neurons, including the downregulation of the agrin gene. We believe that these two states exhibited by these fully differentiated spinal neurons in culture reflect the 

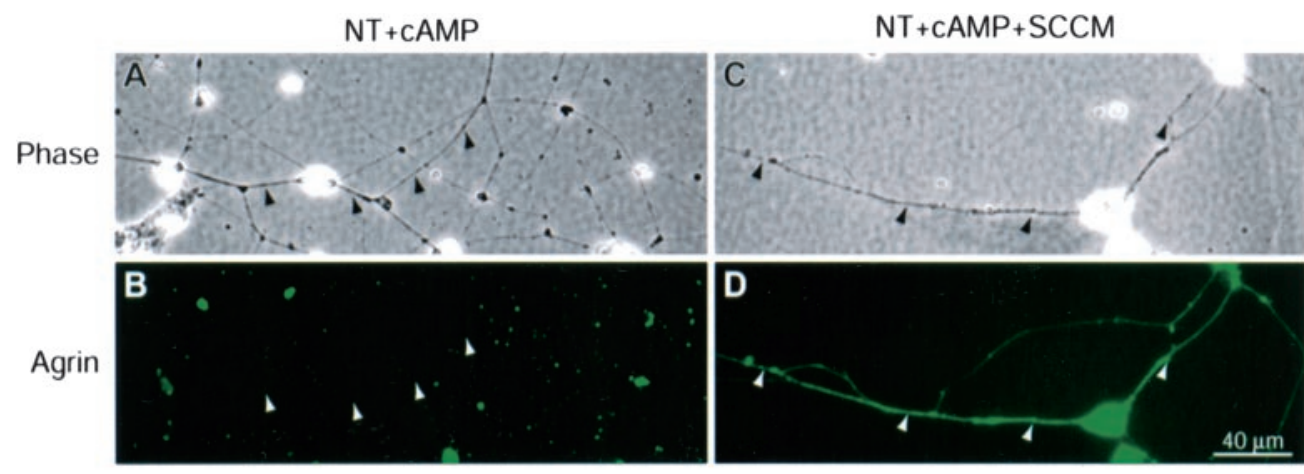

E

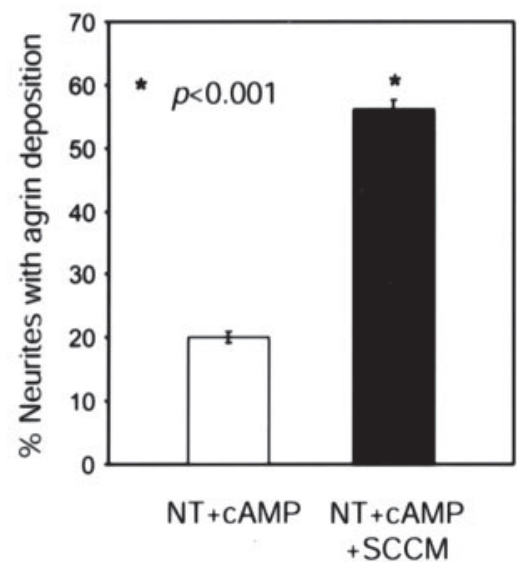

$\mathbf{F}$

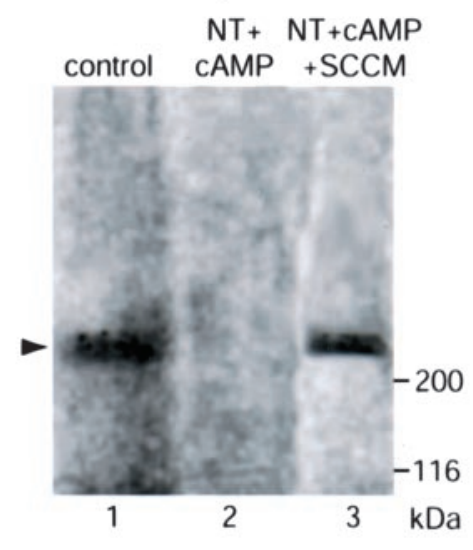

Figure 10. Restoration of agrin deposition and protein level by SCCM. $A, B$, Nerve-muscle cocultures were subject to trophic stimulus (NT + cAMP) only. In this example, no anti-agrin staining ( $B$ ) was found along neurites ( $A, B$, arrowheads). C, D, Nerve-muscle cultures treated with NT plus cAMP plus SCCM showed clear agrin deposition along the neurites (arrowheads). Scale bar: (in $D$ ) $A-D, 40 \mu \mathrm{m}$. These results are quantified in $E$. Only $20.1 \pm 0.9 \%$ of neurites in cocultures with trophic stimulus showed agrin deposition (open bar; 5 experiments). In contrast, ScCM was able to significantly restore this ratio to $56.2 \pm 1.3 \%$ of neurites (filled bar; 5 experiments) (Student's $t$ test; ${ }^{*} p<0.001$ ). $F$, To further confirm this result, agrin protein level in pure neuron cultures was examined by Western blot. In control culture without any treatment, agrin immunoreactivity was detected at above $200 \mathrm{kDa}$ (lane 1, arrowhead). After trophic stimulus, no agrin protein could be detected (lane 2). In contrast, pure neurons treated with NT plus CAMP plus SCCM showed clear agrin immunoreactivity (lane 3). These results suggest that SCCM was able to restore neural agrin expression that was downregulated by NT plus CAMP trophic stimulus, and this regulation was presynaptic.

normal development of motoneurons in vivo. Before coming into contact with the skeletal muscle, trophic and guidance cues allow motoneurons to grow toward the target. Once axons come into contact with the target, they need to switch from a growthoriented state to a synaptogenic state. Our data suggest that environmental cues, including the downregulation of the trophic signals and the presentation of Schwann cell-derived factors, are possible mechanisms for this switch.

\section{Differential effects of trophic stimulation on neuronal survival/growth and synaptogenesis}

During normal NMJ development in vivo, once motor axons come into contact with muscle fibers, they induce the formation of AChR clusters (Anderson et al., 1977; Frank and Fischbach, 1979; Sanes and Lichtman, 1999). This postsynaptic differentiation is accompanied by the cessation of axonal growth and the initiation of presynaptic differentiation, leading to the establishment of a stable junctional complex. The axons terminate near the main intramuscular nerve in an end-plate band (Sanes and Lichtman, 2001). When NMJ formation is disrupted in animals with targeted deletion in agrin or MuSK genes, motor axons do not terminate near the main intramuscular nerve but continue to grow throughout the muscle fiber (DeChiara et al., 1996; Gautam et al., 1996). This enhanced growth of motor axons in these NMJdeficient animals bears resemblance to the situation in our nerve-muscle cocultures under the influence of trophic stimuli as shown here. Our results thus suggest the possibility that, in the absence of NMJ formation in agrin and MuSK knock-out animals, motor axons experience a higher level of trophic stimuli that are globally distributed in the target muscle field.

Previous studies have suggested that the skeletal muscle can regulate its trophic influence on motoneurons on the basis of NMJ function. For example, the programmed cell death of spinal motoneurons during normal embryogenesis is reduced when the muscle is chronically treated with activity blockers such as curare (Pittman and Oppenheim, 1978; Houenou et al., 1991; Usiak and Landmesser, 1999). This may be attributable to the elevation of the level of trophic factors as a result of NMJ blockade. In addition to the increased axonal growth along the muscle fiber in agrin, rapsyn, and MuSK knock-out animals showing NMJ deficiency, the survival of motoneurons is actually enhanced (Terrado et al., 2001). All of these results are consistent with the notion that aberrant NMJ development leads to an increase in target-derived trophic stimuli that sustain enhanced motoneuron survival and growth. It follows that normal NMJ formation is necessary for the downregulation of trophic stimulation on motor axons to establish stable nerve-muscle contacts and synaptogenesis. As the NMJ is established, there may be a decrease in the global presence of trophic signals and an increase in their local concentration at the developing junction. Although it is not 
known whether neurotrophins are localized at the NMJ, the TrkB receptor is indeed concentrated at the postsynaptic membrane (Gonzalez et al., 1999). The localization of trophic stimuli is probably necessary for the stabilization of the nerve-muscle contact. Thus, in addition to AChR clustering, the postsynaptic differentiation may also involve the redistribution of trophic stimuli to the subsynaptic site. There appears to be a reciprocal signaling between the innervating nerve and its target muscle. First, trophic stimuli promote the growth and branching of motor axons during the early stages of innervation. We speculate that, once AChR clusters are induced, a global downregulation and a synapsespecific localization of trophic molecules take place to allow stabilization of the nerve-muscle contact and the survival of motoneurons that successfully establish their NMJs. In addition to trophic effects on promoting neuronal survival, neurotrophins are also known to increase the secretion of neurotransmitters at the NMJ as described above (Lohof et al., 1993; He et al., 2000; Poo, 2001). Thus, the localization of trophic stimuli at the NMJ serves multiple functions in stabilizing the nerve-muscle contact, providing trophic support to motoneurons that successfully establish NMJs and a mechanism to increase the efficacy of synaptic transmission. In addition to its effect on postsynaptic AChR clustering, our preliminary study also indicates that trophic stimulation via neurotrophins and cAMP downregulates presynaptic differentiation as shown by a decrease in the number of varicosities along the spinal neurite in culture (H. W. Hung and H. B. Peng, unpublished observations).

A previous study showed that BDNF and NT-4 also have a postsynaptic effect on AChR clustering induced by agrin (Wells et al., 1999). At low agrin concentration, AChR clustering is inhibited by these two neurotrophins but not by NGF or NT-3 in cultured chick myotubes. The inhibition is overcome when the agrin concentration is high. Our study with Xenopus muscle cultures, however, has not shown a postsynaptic neurotrophin effect (Fig. 5). Neither bead nor agrin-induced AChR clustering was inhibited by neurotrophins up to a concentration of $100 \mathrm{ng} / \mathrm{ml}$, whereupon their effect on chick myotubes was obvious. A possible explanation for this discrepancy is that, unlike the chick myotube, the Xenopus muscle cell may lack or have very low TrkB receptor expression. However, our results have clearly shown that neurotrophins act on the nerve terminal to inhibit innervationinduced AChR clustering.

\section{Differential regulation of agrin expression by neurotrophins and Schwann cell-derived factor(s)}

Although it has been well documented that agrin plays a major role in the formation of the NMJ (Bowe and Fallon, 1995; Sanes and Lichtman, 2001), remarkably little is known about how the expression of agrin genes and proteins are regulated. It has been shown that cultured Xenopus spinal neurons deposit agrin along the neuritic track (Cohen et al., 1994, 1995). Consistent with these findings, our data also showed agrin association with both large and small neuritic branches in control cultures. Thus, agrin deposition is probably a constitutive process in vitro. As shown in the present study, both the synthesis and deposition of agrin can in fact be downregulated by neurotrophins. The downregulation is a direct effect on the neuron rather than on the muscle. Thus, it is most likely that the decrease in agrin expression and deposition accounts for the lack of synaptogenic activity of trophic factorstimulated neurons. The present study has also shown for the first time that glial cells play a role in the regulation of neuronal agrin gene expression. SCCM can reverse the inhibition of agrin gene expression by the trophic stimulation in vitro. Similar to neuro- trophins, SCCM directly affects the agrin gene expression (Fig. $8 \mathrm{~B}$ ), protein level (Fig. $10 \mathrm{~F}$ ), and deposition (Fig. 10A-E) in neurons. Our recent study has shown that Schwann cells themselves are also capable of synthesizing agrin, including both active and nonactive forms (Yang et al., 2001). Thus, it is possible that agrin provided by SCCM might affect postsynaptic differentiation at nerve-muscle contacts under trophic stimulation. This is, however, unlikely because AChR clusters conform to the nervemuscle contacts rather than scatter over the entire muscle cell as in the situation of bath application of agrin to cultured muscle cells. Furthermore, although cultured Schwann cells may enhance AChR aggregation through the continuous release of native agrin protein, the SCCM by itself does not increase AChR clustering (Yang et al., 2001). This is probably attributable to the fact that the amount of active agrin is low in SCCM. Thus, the restoration of agrin expression and enhanced synaptogenesis by SCCM likely results from a direct effect on the neurons by some unknown factor(s) released by Schwann cells.

\section{The role of glial cells in synaptogenesis}

The NMJ is composed of the presynaptic nerve terminal, postsynaptic membrane specializations, and synapse-associated glial cells called perisynaptic Schwann cells (PSCs). Recent studies have shown the pivotal role of PSCs in leading the growth of the nerve terminal at the NMJ during synaptic sprouting and development (Son and Thompson, 1995a,b; Trachtenberg and Thompson, 1997; Herrera et al., 2000; Koirala et al., 2000). In addition, they also lead the way in guiding nerve terminal sprouting when neurotransmission is interrupted (Son and Thompson, 1995b) and during synaptic remodeling (Ko and Chen, 1996). Our current data suggest a new role of Schwann cells in regulating the switching from the growth to synaptogenic state of motoneurons. Although the molecular mechanism of how glial cells promote synaptic growth is not known, the present study on the effects of SCCM suggests that diffusible factor(s) secreted by Schwann cells promote the synaptogenic potential of trophic factor-stimulated axons. Our recent finding has also shown that SCCM greatly increases transmitter release at nerve-muscle contacts in Xenopus cultures (Cao and Ko, 2001). These observations are consistent with the recent findings that glia-derived factors enhance synaptogenesis and transmitter release in CNS neuron cultures (Pfrieger and Barres, 1997; Mauch et al., 2001; Nagler et al., 2001). Thus, glial cells may play multiple roles in promoting synaptic growth and function during synaptogenesis. On the basis of previous studies and the current findings, we propose the following sequence of events during $\mathrm{NMJ}$ formation in vivo. Before innervation, there is a global expression of trophic signals in the target muscle that allow motor axons to grow on its surface. Their contact with perisynaptic Schwann cells causes a switch of the growth state into synaptogenic state that allows the induction of NMJ formation. This causes the muscle cell to downregulate the trophic stimuli and to localize them at the NMJ.

Schwann cells are known to release various growth factors, such as neurotrophin 3, insulin-like growth factor, as well as extracellular matrix molecules (Mirsky et al., 2002). However, it is not known whether and how any of these or novel factors derived from Schwann cells may be responsible for the upregulation of neuronal agrin expression and the enhancement of synapse formation after the neurotrophic stimulation. One possible mechanism for the upregulation is that Schwann cell-derived factors may inhibit the level of cAMP and/or the downstream effects of neurotrophic stimulation in motor neurons, thereby preventing the downregulation of agrin expression in neurons 
treated with neurotrophins. This explanation would be consistent with our finding that SCCM treatment by itself is not effective in promoting agrin expression in motor neurons when they are not under the trophic stimulation. The identification of Schwann cell-derived signals and how these signals interplay with neurotrophins/cAMP in promoting neuronal agrin expression and synaptogenesis are now under investigation. Future studies aimed at understanding the regulation of the synthesis and redistribution of trophic factors by the muscle and the role of Schwann cells in promoting the synaptogenic fate of the motoneuron should lead to additional advances in our understanding of NMJ development.

\section{References}

Anderson MJ, Cohen MW (1977) Nerve-induced and spontaneous redistribution of acetylcholine receptors on cultured muscle cells. J Physiol (Lond) 268:757-773.

Anderson MJ, Cohen MW, Zorychta E (1977) Effects of innervation on the distribution of acetylcholine receptors on cultured muscle cells. J Physiol (Lond) 268:731-756.

Becker E, Soler RM, Yuste VJ, Gine E, Sanz-Rodriguez C, Egea J, MartinZanca D, Comella JX (1998) Development of survival responsiveness to brain-derived neurotrophic factor, neurotrophin 3 and neurotrophin $4 / 5$, but not to nerve growth factor, in cultured motoneurons from chick embryo spinal cord. J Neurosci 18:7903-7911.

Bowe MA, Fallon JR (1995) The role of agrin in synapse formation. Annu Rev Neurosci 18:443-462.

Cao G, Ko C-P (2001) Schwann cell-conditioned medium modulates synaptic activities at Xenopus neuromuscular junctions in vitro. Soc Neurosci Abstr 27:711.12.

Carmignoto G, Pizzorusso T, Tia S, Vicini S (1997) Brain-derived neurotrophic factor and nerve growth factor potentiate excitatory synaptic transmission in the rat visual cortex. J Physiol (Lond) 498:153-164.

Cohen MW (1980) Development of an amphibian neuromuscular junction in vivo and in culture. J Exp Biol 89:43-56.

Cohen MW, Moody-Corbett F, Godfrey EW (1994) Neurite deposition of agrin on culture substrate: implication for nerve-muscle synaptogenesis. J Neurosci 14:3293-3303.

Cohen MW, Moody-Corbett F, Godfrey EW (1995) Former neuritic pathways containing endogenous neural agrin have high synaptogenic activity. Dev Biol 167:458-468.

Daggett DF, Stone D, Peng HB, Nikolics K (1996) Full-length agrin isoform activities and binding site distributions on cultured Xenopus muscle cells. Mol Cell Neurosci 7:75-88.

Dai Z, Peng HB (1998) A role of tyrosine phosphatase in acetylcholine receptor cluster dispersal and formation. J Cell Biol 141:1613-1624.

Davies AM (1994) The role of neurotrophins in the developing nervous system. J Neurobiol 25:1334-1348.

DeChiara TM, Bowen DC, Valenzuela DM, Simmons MV, Poueymirou WT, Thomas S, Kinetz E, Compton DL, Rojas E, Park JS, Smith C, DiStefano PS, Glass DJ, Burden SJ, Yancopoulos GD (1996) The receptor tyrosine kinase MuSK is required for neuromuscular junction formation in vivo. Cell 85:501-512.

Frank E, Fischbach GD (1979) Early events in neuromuscular junction formation in vitro. J Cell Biol 83:143-158.

Funakoshi H, Belluardo N, Arenas E, Yamamoto Y, Casabona A, Persson H, Ibáñez CF (1995) Muscle-derived neurotrophin-4 as an activity-dependent trophic signal for adult motor neurons. Science 268:1495-1499.

Gautam M, Noakes PG, Moscoso L, Rupp F, Scheller RH, Merlie JP, Sanes JR (1996) Defective neuromuscular synaptogenesis in agrin-deficient mutant mice. Cell 85:525-535.

Gesemann M, Denzer AJ, Ruegg MA (1995) Acetylcholine receptoraggregating activity of agrin isoforms and mapping of the active site. J Cell Biol 128:625-636.

Glass DJ, Bowen DC, Stitt TN, Radziejewski C, Bruno J, Ryan TE, Gies DR, Shah S, Mattsson K, Burden SJ, DiStefano PS, Valenzuela DM, DeChiara TM, Yancopoulos GD (1996) Agrin acts via a MuSK receptor complex. Cell 85:513-523.

Godfrey EW (1991) Comparison of agrin-like proteins from the extracellular matrix of chicken kidney and muscle with neural agrin, a synapse organizing protein. Exp Cell Res 195:99-109.
Gonzalez M, Ruggiero FP, Chang Q, Shi YJ, Rich MM, Kraner S, BaliceGordon RJ (1999) Disruption of TrkB-mediated signaling induces disassembly of postsynaptic receptor clusters at neuromuscular junctions. Neuron 24:567-583.

Hanson Jr MG, Shen S, Wiemelt AP, McMorris FA, Barres BA (1998) Cyclic AMP elevation is sufficient to promote the survival of spinal motor neurons in vitro. J Neurosci 18:7361-7371.

He X, Yang F, Xie Z, Lu B (2000) Intracellular $\mathrm{Ca}^{2+}$ and $\mathrm{Ca}^{2+} /$ calmodulindependent kinase II mediate acute potentiation of neurotransmitter release by neurotrophin-3. J Cell Biol 149:783-792.

Henderson CE, Camu W, Mettling C, Gouin A, Poulsen K, Karihaloo M, Rullamas J, Evans T, McMahon SB, Armanini MP, Berkemeier L, Phillips HS, Rosenthal A (1993) Neurotrophins promote motor neuron survival and are present in embryonic limb bud. Nature 363:266-270.

Henderson CE, Phillips HS, Pollock RA, Davies AM, Lemeulle C, Armanini M, Simpson LC, Moffet B, Vandlen RA, Koliatsos VE, Rosenthal A (1994) GDNF: a potent survival factor for motoneurons present in peripheral nerve and muscle. Science 266:1062-1064.

Herrera AA, Qiang H, Ko CP (2000) The role of perisynaptic Schwann cells in development of neuromuscular junctions in the frog (Xenopus laevis). J Neurobiol 45:237-254.

Houenou LJ, McManaman JL, Prevette D, Oppenheim RW (1991) Regulation of putative muscle-derived neurotrophic factors by muscle activity and innervation: in vivo and in vitro studies. J Neurosci 11:2829-2837.

Hughes RA, Sendtner M, Thoenen H (1993) Members of several gene families influence survival of rat motoneurons in vitro and in vivo. J Neurosci Res 36:663-671.

Klintsova AY, Greenough WT (1999) Synaptic plasticity in cortical systems. Curr Opin Neurobiol 9:203-208.

Ko CP, Chen L (1996) Synaptic remodeling revealed by repeated in vivo observations and electron microscopy of identified frog neuromuscular junctions. J Neurosci 16:1780-1790.

Koirala S, Qiang H, Ko CP (2000) Reciprocal interactions between perisynaptic Schwann cells and regenerating nerve terminals at the frog neuromuscular junction. J Neurobiol 44:343-360.

Koliatsos VE, Clatterbuck RE, Winslow JW, Cayouette MH, Price DL (1993) Evidence that brain-derived neurotrophic factor is a trophic factor for motor neurons in vivo. Neuron 10:359-367.

Kuromi H, Kidokoro Y (1984) Nerve disperses preexisting acetylcholine receptor clusters prior to induction of receptor. Dev Biol 103:53-61.

Leonard DG, Gorham JD, Cole P, Greene LA, Ziff EB (1988) A nerve growth factor-regulated messenger RNA encodes a new intermediate filament protein. J Cell Biol 106:181-193.

Lohof AM, Ip NY, Poo M (1993) Potentiation of developing neuromuscular synapses by the neurotrophins NT-3 and BDNF. Nature 363:350-353.

Maisonpierre PC, Belluscio L, Friedman B, Alderson RF, Wiegand SJ, Furth ME, Lindsay RM, Yancopoulos GD (1990) NT-3, BDNF, and NGF in the developing rat nervous system: parallel as well as reciprocal patterns of expression. Neuron 5:501-509.

Mauch DH, Nagler K, Schumacher S, Goritz C, Muller EC, Otto A, Pfrieger FW (2001) CNS synaptogenesis promoted by glia-derived cholesterol. Science 294:1354-1357.

McMahan UJ (1990) The agrin hypothesis. Cold Spring Harbor Symp Quant Biol 55:407-418.

McMahan UJ, Horton SE, Werle MJ, Honig LS, Kroger S, Ruegg MA, Escher G (1992) Agrin isoforms and their role in synaptogenesis. Curr Opin Cell Biol 4:869-874.

Ming G, Lohof AM, Zheng JQ (1997) Acute morphogenic and chemotropic effects of neurotrophins on cultured embryonic Xenopus spinal neurons. J Neurosci 17:7860-7871.

Mirsky R, Jessen KR, Brennan A, Parkinson D, Dong Z, Meier C, Parmantier E, Lawson D (2002) Schwann cells as regulators of nerve development. J Physiol (Paris) 96:17-24.

Moody-Corbett F, Cohen MW (1982) Influence of nerve on the formation and survival of acetylcholine receptor and cholinesterase patches on embryonic Xenopus muscle cells in culture. J Neurosci 2:633-646.

Nagler K, Mauch DH, Pfrieger FW (2001) Glia-derived signals induce synapse formation in neurones of the rat central nervous system. J Physiol (Lond) 533:665-679.

Peng HB, Baker LP, Chen Q (1991) Tissue culture of Xenopus neurons and muscle cells as a model for studying synaptic induction. In: Methods in 
cell biology, Vol 36 (Kay BK, Peng HB, eds), pp 511-526. San Diego: Academic.

Peng HB, Ali AA, Dai Z, Daggett DF, Raulo E, Rauvala H (1995) The role of heparin-binding growth-associated molecule (HB-GAM) in the postsynaptic induction in cultured muscle cells. J Neurosci 15:3027-3038.

Pfrieger FW, Barres BA (1997) Synaptic efficacy enhanced by glial cells in vitro. Science 277:1684-1687.

Pittman RH, Oppenheim RW (1978) Neuromuscular blockade increases motoneurone survival during normal cell death in the chick embryo. Nature 271:364-366.

Poo MM (2001) Neurotrophins as synaptic modulators. Nat Rev Neurosci 2:24-32.

Sanes JR, Lichtman JW (1999) Development of the vertebrate neuromuscular junction. Annu Rev Neurosci 22:389-442.

Sanes JR, Lichtman JW (2001) Induction, assembly, maturation and maintenance of a postsynaptic apparatus. Nat Rev Neurosci 2:791-805.

Seil FJ, Drake-Baumann R (2000) TrkB receptor ligands promote activitydependent inhibitory synaptogenesis. J Neurosci 20:5367-5373.

Snider WD (1994) Functions of the neurotrophins during nervous system development: what the knockouts are teaching us? Cell 77:627-638.

Son Y-J, Thompson WJ (1995a) Schwann cell processes guide regeneration of peripheral axons. Neuron 14:125-132.

Son Y-J, Thompson WJ (1995b) Nerve sprouting in muscle is induced and guided by processes extended by Schwann cells. Neuron 14:133-141.

Son Y-J, Trachtenberg JT, Thompson WJ (1996) Schwann cells induce and guide sprouting and reinnervation of neuromuscular junctions. Trends Neurosci 19:280-285.

Song HJ, Poo MM (1999) Signal transduction underlying growth cone guidance by diffusible factors. Curr Opin Neurobiol 9:355-363.

Song H, Poo M (2001) The cell biology of neuronal navigation. Nat Cell Biol 3:E81-E88.

Terrado J, Burgess RW, DeChiara T, Yancopoulos G, Sanes JR, Kato AC
(2001) Motoneuron survival is enhanced in the absence of neuromuscular junction formation in embryos. J Neurosci 21:3144-3150.

Thoenen H (1995) Neurotrophins and neuronal plasticity. Science 270:593-598.

Trachtenberg JT, Thompson WJ (1997) Nerve terminal withdrawal from rat neuromuscular junctions induced by neuregulin and Schwann cells. J Neurosci 17:6243-6255.

Tsim KWK, Ruegg MA, Escher G, Kröger S, McMahan UJ (1992) cDNA that encodes active agrin. Neuron 8:677-689.

Unsicker K, Krisch B, Otten U, Thoenen H (1978) Nerve growth factorinduced fiber outgrowth from isolated rat adrenal chromaffin cells: impairment by glucocorticoids. Proc Natl Acad Sci USA 75:3498-3502.

Usiak MF, Landmesser LT (1999) Neuromuscular activity blockade induced by muscimol and $d$-tubocurarine differentially affects the survival of embryonic chick motoneurons. J Neurosci 19:7925-7939.

Vejsada R, Sagot Y, Kato AC (1995) Quantitative comparison of the transient rescue effects of neurotrophic factors on axotomized motoneurons in vivo. Eur J Neurosci 7:108-115.

Wang T, Xie K, Lu B (1995) Neurotrophins promote maturation of developing neuromuscular synapses. J Neurosci 15:4796-4805.

Wang CY, Yang F, He XP, Je HS, Zhou JZ, Eckermann K, Kawamura D, Feng L, Shen L, Lu B (2002) Regulation of neuromuscular synapse development by glial cell line-derived neurotrophic factor and neurturin. J Biol Chem 277:10614-10625.

Wells DG, McKechnie BA, Kelkar S, Fallon JR (1999) Neurotrophins regulate agrin-induced postsynaptic differentiation. Proc Natl Acad Sci USA 96:1112-1117.

Yang JF, Cao G, Koirala S, Reddy LV, Ko CP (2001) Schwann cells express active agrin and enhance aggregation of acetylcholine receptors on muscle fibers. J Neurosci 21:9572-9584.

Zigmond MJ, Bloom FE, Landis SC, Roberts JL, Squire LR (1999) Fundamental neuroscience, pp 519-635. San Diego: Academic Press. 